\title{
Hydroxytyrosol improves mitochondrial function and reduces oxidative stress in the brain of $d b / d b$ mice: role of AMP-activated protein kinase activation
}

\author{
Adi Zheng ${ }^{1}$, Hao $\mathrm{Li}^{1}$, Jie $\mathrm{Xu}^{1}$, Ke $\mathrm{CaO}^{2}$, Hua $\mathrm{Li}^{1}$, Wenjun $\mathrm{Pu}^{1}$, Ziqi Yang ${ }^{1}$, Yunhua Peng ${ }^{2}$, \\ Jiangang Long ${ }^{1}$, Jiankang Liu ${ }^{1 *}$ and Zhihui Feng ${ }^{2 *}$ \\ ${ }^{1}$ The Key Laboratory of Biomedical Information Engineering of Ministry of Education, School of Life Science and Technology, \\ Xi'an Jiaotong University, Xi'an 710049, People's Republic of China \\ ${ }^{2}$ Center for Mitochondrial Biology and Medicine, Frontier Institute of Science and Technology, Xi'an Jiaotong University, \\ 28 West Xianning Road, Xi'an 710049, People's Republic of China
}

(Submitted 3 October 2014 - Final revision received 13 February 2015 - Accepted 2 March 2015 - First published online 17 April 2015)

\section{Abstract}

Hydroxytyrosol (HT) is a major polyphenolic compound found in olive oil with reported anti-cancer and anti-inflammatory activities. However, the neuroprotective effect of HT on type 2 diabetes remains unknown. In the present study, $d b / d b$ mice and SH-SY-5Y neuroblastoma cells were used to evaluate the neuroprotective effects of HT. After 8 weeks of HT administration at doses of 10 and $50 \mathrm{mg} / \mathrm{kg}$, expression levels of the mitochondrial respiratory chain complexes I/II/IV and the activity of complex I were significantly elevated in the brain of $d b / d b$ mice. Likewise, targets of the antioxidative transcription factor nuclear factor erythroid 2 related factor 2 including p62 (sequestosome-1), haeme oxygenase 1 (HO-1), and superoxide dismutases 1 and 2 increased, and protein oxidation significantly decreased. HT treatment was also found to activate AMP-activated protein kinase (AMPK), sirtuin 1 and PPAR $\gamma$ coactivator- $1 \alpha$, which constitute an energy-sensing protein network known to regulate mitochondrial function and oxidative stress responses. Meanwhile, neuronal survival indicated by neuron marker expression levels including activity-regulated cytoskeleton-associated protein, $N$-methyl-D-aspartate receptor and nerve growth factor was significantly improved by HT administration. Additionally, in a high glucose-induced neuronal cell damage model, HT effectively increased mitochondrial complex IV and HO-1 expression through activating AMPK pathway, followed by the prevention of high glucose-induced production of reactive oxygen species and declines of cell viability and $\mathrm{V}_{\mathrm{O} 2}$ capacity. Our observations suggest that $\mathrm{HT}$ improves mitochondrial function and reduces oxidative stress potentially through activation of the AMPK pathway in the brain of $d b / d b$ mice.

\section{Key words: Hydroxytyrosol: $\boldsymbol{d} b / \boldsymbol{d b}$ Mice: Mitochondrial function: Oxidative stress: AMP-activated protein kinase pathway: Reactive oxygen species}

The escalating epidemic of the metabolic syndromes, including obesity and diabetes, represents one of the most pressing and costly medical challenges in public health of the twentyfirst century. A recent study has reported that 336 million people had diabetes in 2011, and this number is expected to rise to 552 million by $2030^{(1)}$. Diabetes is recognised by hyperglycaemia and glucose intolerance due to insulin deficiency, impaired insulin sensitivity or both. Type 2 diabetes mellitus (T2DM) is predominant and accounts for $90 \%$ of patients with diabetes ${ }^{(2)}$. It is well established that diabetes has been associated with slowly progressing brain damage that impaired cognitive function ${ }^{(3-5)}$. Although there is some evidence for a relationship between T2DM and brain damage, the mechanisms driving it remain unknown.

Clinical studies have indicated that cognitive dysfunction is correlated with brain atrophy in patients with diabetes ${ }^{(3,6,7)}$. Kumar et $a{ }^{\left({ }^{(8)}\right.}$ reported smaller total brain grey matter volumes in patients with diabetes, and the decrease is more pronounced in the cortical grey matter of the temporal lobe ${ }^{(9)}$. Despite the limited knowledge on the underlying mechanisms for cognitive dysfunction during the progression of T2DM, mitochondrial dysfunction and oxidative stress have been suggested as major contributors ${ }^{(10-12)}$. A previous study has indicated that high glucose concentration, a major pathological characteristic of diabetes, could induce

Abbreviations: AMPK, AMP-activated protein kinase; Arc, activity-regulated cytoskeleton-associated protein; HO-1, haeme oxygenase 1; HT, hydroxytyrosol; NGF, nerve growth factor; NMDAR1, $N$-methyl-D-aspartate receptor 1 ; Nrf2, nuclear transcription factor erythroid 2p45-related factor-2; p-AMPK, phosphoAMPK; PGC-1, PPAR $\gamma$ coactivator-1; ROS, reactive oxygen species; Sirt1, sirtuin 1; SOD, superoxide dismutase; T2DM, type 2 diabetes mellitus; TBST, Tris-buffered saline Tween 20 .

*Corresponding authors: J. Liu, fax +8629 82665849, email j.liu@mail.xjtu.edu.cn; Z. Feng, fax +86 2982665849 , email zhfeng@mail.xjtu.edu.cn 
overproduction of superoxide from mitochondrial electron transport chain, which is considered the first and key event involved in the pathogenesis of diabetic and subsequent complications $^{(13)}$. Overexpression of Mn superoxide dismutase (SOD) 2 protects neurons against hyperglycaemic injury in $d b / d b$ diabetic mice ${ }^{(14)}$. It has also been reported that high glucose concentrations could enhance the formation of advanced glycation end products, which can induce reactive oxygen species (ROS) production from the mitochondria and promote oxidative damage to the heart and brain ${ }^{(15,16)}$. Given the importance of the mitochondria as a source of both ATP and ROS, mitochondrial dysfunction has been considered a link between diabetes and neurodegenerative diseases such as Alzheimer's disease ${ }^{(17)}$. While numerous mechanisms regulating mitochondrial function have been delineated, including mitochondrial biogenesis, dynamics, modification and mitophagy, the clear mechanisms accounting for mitochondrial dysfunction during T2DM-associated brain damage remain to be elucidated.

Hydroxytyrosol (HT), a natural polyphenol from virgin olive oil, is considered to be one of the most effective antioxidants. Consumption of HT has certain health benefits, and the responsible mechanisms for these effects have been mainly attributed to its ability to scavenge ROS and enhance endogenous antioxidant systems ${ }^{(18,19)}$. In our previous studies, we have found that HT could protect retinal epithelial pigment cells and adipocytes against oxidative damage through activating the nuclear transcription factor erythroid 2p45-related factor-2 (Nrf2)/Kelch-like ECH-associated protein 1 pathway ${ }^{(20-22)}$ and stimulate mitochondrial biogenesis through PPAR $\gamma$ coactivator-1 (PGC-1) $\alpha$ activation ${ }^{(20,23)}$. These studies have suggested that HT possesses the ability to reduce oxidative stress and improve mitochondrial function. However, the beneficial effects of HT on diabetic brain damage remain unknown. Thus, because HT was reported to efficiently cross the blood-brain barrier ${ }^{(24)}, d b / d b$ mice, a widely used human T2DM animal model, were used to explore the in vivo neuroprotective effects of HT and its underlying mechanisms.

\section{Experimental methods}

\section{Chemicals}

An antibody against $\beta$-actin was obtained from Sigma. Antibodies against complexes I (NADH dehydrogenase (ubiquinone) Fe-S protein 3; NDUFS3), II (subunit $30 \mathrm{kDa}$ ), III (subunit core 2), IV (subunit I) and V (subunit $\alpha$ ) were obtained from Invitrogen. Antibodies against AMP-activated protein kinase (AMPK), phospho-AMPK (p-AMPK) and PGC-1 were obtained from Cell Signaling Technology. Antibodies against sirtuin 1 (Sirt1), p62 (sequestosome-1), haeme oxygenase 1 (HO-1), SOD1 and SOD2 were obtained from Santa Cruz Biotech. The BCA Protein Assay kit (Pierce 23225) and enhanced chemiluminescence (ECL) Western blotting detection kit were purchased from Pierce. Nitrocellulose membranes were obtained from PerkinElmer Life Sciences. The TRIzol reagent, oligomycin, carbonyl cyanide 4-(trifluoromethoxy) phenylhydrazone (FCCP), rotenone and compound $\mathrm{C}$ were obtained from Invitrogen, and the immunoprecipitation lysis buffer was from Beyotime. HT was purchased from Xi'an APP-Chem Bio(Tech) Company Limited.

\section{Animals and treatments}

Male $d b / d b$ mice aged 4-week-old from a C57BL/6J genetic background were purchased from SLAC Laboratory Animals Company Limited. After 1 week of acclimatisation, mice were randomly divided into the following three groups: $d b / d b$ mice; $d b / d b$ mice with a daily oral administration of low-dose HT (10 $\mathrm{mg} / \mathrm{kg}$ per $\mathrm{d}) ; d b / d b$ mice with a daily oral administration of high-dose HT $(50 \mathrm{mg} / \mathrm{kg}$ per d). After 8 weeks of feeding, mice were fasted overnight and killed. All animals were housed in a temperature-controlled $\left(25-27^{\circ} \mathrm{C}\right)$ and humiditycontrolled (60\%) animal room and maintained on a $12 \mathrm{~h}$ light $-12 \mathrm{~h}$ dark cycle (light from 08.00 to 20.00 hours) with food and water provided during the experiments. All the procedures were performed in accordance with the United States Public Health Services Guide for the Care and Use of Laboratory Animals, and all efforts were made to minimise the suffering and the number of animals used in the present study.

\section{Western blotting}

Cortical brain tissue was lysed with Western and immunoprecipitation lysis buffer. Lysates were centrifuged at $13000 \boldsymbol{g}$ for $10 \mathrm{~min}$ at $4^{\circ} \mathrm{C}$. Supernatant protein concentrations were determined with a BCA protein assay kit (Pierce). Equal amounts of protein per sample $(20 \mu \mathrm{g})$ were subjected to $10 \%(\mathrm{w} / \mathrm{v})$ SDSPAGE; proteins were then transferred to nitrocellulose membranes and blocked with a $5 \%(\mathrm{w} / \mathrm{v})$ non-fat milk/TBST (Tris-buffered saline Tween 20) for $1 \mathrm{~h}$ at room temperature. Membranes were incubated with primary antibodies against $\beta$-actin (1:5000), mitochondrial complex I-V (1:3000), AMPK (1:1000), phospho-AMPK (1:1000), PGC-1 (1:1000), Sirt1 (1:1000), p62 (1:1000), HO-1 (1:1000), SOD1 (1:1000) and SOD2 (1:1000) in 5\% (w/v) milk/TBST at $4^{\circ} \mathrm{C}$ overnight. Membranes were washed with TBST three times and were then incubated with anti-rabbit or anti-mouse antibodies at room temperature for $1 \mathrm{~h}$. Chemiluminescent detection was performed by an ECL Western blotting detection kit (Pierce) and quantified by scanning densitometry.

\section{Real-time $P C R$}

Total RNA was extracted from cells using TRIzol reagent according to the manufacturer's instructions. Reverse transcription from RNA to complementary DNA was performed using the PrimeScript RT-PCR Kit (Takara) followed by semiquantitative real-time PCR using gene-specific primers. The following primers were used for the RT-PCR analysis: activity-regulated cytoskeleton-associated protein ( $A r c$ ), GGTAAGTGCCGAGCTGAGATG (forward) and CGACCTGTGCAACCCTTTC (reverse); $N$-methyl-D-aspartate receptor 1 (NMDAR1), GCCCAACGCCATACAGATG (forward) and GGCGGGTGACTAACTAGGATAGC (reverse); nerve growth factor $(N G F)$, TTGCCAAGGACGCAGCTTT (forward) and TGCCTGTACGCCGATCAAA 
(reverse); $\beta$-actin, CCACACCTTCTACAATGAGC (forward) and GGTCTCAAACATGATCTGGG (reverse).

\section{Assays for mitochondrial complex activities}

Mitochondria were isolated from mouse cortical brain tissue, and mitochondrial protein concentrations were determined using the BCA protein assay kit (Pierce). NADH-ubiquinone reductase (complex I) activities were measured spectrometrically using conventional assays, as described previously ${ }^{(25)}$.

\section{Cell viability assay}

SH-SY-5Y cells were seeded in ninety-six-well plates at a density of $4 \times 10^{4}$ cells per well for $24 \mathrm{~h}$. These cells were then treated with different concentrations of glucose or HT for the indicated time periods. Then, the number of viable cells was determined by addition of 3-(4,5-dimethylthiazol-2-yl)2,5-diphenyltetrazolium bromide. Optical densities were read at $550 \mathrm{~nm}$ using a microplate spectrophotometer (Multiskan Ascent; Thermo Fisher Scientific, Inc.).

\section{Oxidative status assessment}

$2^{\prime}, 7^{\prime}$-Dichlorodihydrofluorescein diacetate $\left(2^{\prime}, 7^{\prime}\right.$-dichlorofluorescin diacetate) is a freely permeable fluorogenic tracer used for the assessment of oxidative status. After treatment, cells were incubated with $10 \mathrm{~mm}-2^{\prime}, 7^{\prime}$-dichlorofluorescin diacetate for $30 \mathrm{~min}$ and then washed with PBS three times. Cell lysis was prepared with a lysis solution $(10 \mathrm{~mm}$-Tris, $150 \mathrm{~mm}-\mathrm{NaCl}$, $0.1 \mathrm{~mm}$-EDTA and $0.5 \%$ Triton X-100, pH 7.5). The supernatant $(200 \mu \mathrm{l})$ was analysed using a spectrofluorometer with excitation $485 \mathrm{~nm}$ and emission $538 \mathrm{~nm}$ (Fluoroskan Ascent; Thermo Fisher Scientific, Inc.). An aliquot of supernatant was used in a BCA protein assay to determine the concentration of total protein. ROS levels were expressed as the relative 2,7-dichlorofluorescien (DCF) fluorescence per $\mu \mathrm{g}$ of protein.

$\mathrm{V}_{\mathrm{O} 2}$

Cell $\mathrm{V}_{\mathrm{O} 2}$ by intact cells, which is a marker for mitochondrial respiration activity, was measured. Basal, oligomycin and FCCP-treated $\mathrm{V}_{\mathrm{O} 2}$ rates were investigated after high glucose or HT treatment using Seahorse Extracellular Flux Analyzer (Seahorse Bioscience) according to the manufacturer's instructions.

\section{Statistical analysis}

Data are presented as means with their standard errors. The statistical significance of differences among groups were analysed using a one-way ANOVA followed by Dunnett's multiple comparison test. A $P$ value $<0.05$ was considered to be statistically significant. (a)

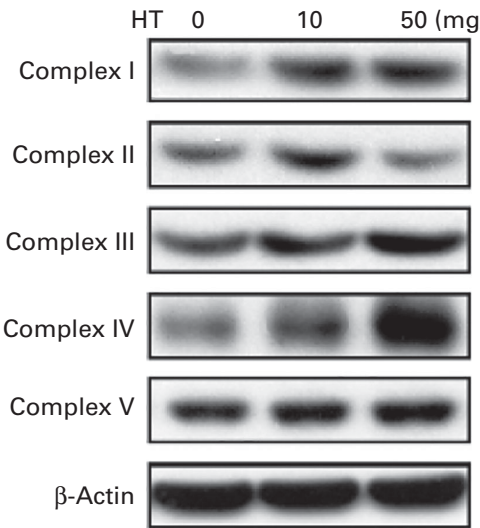

(c)

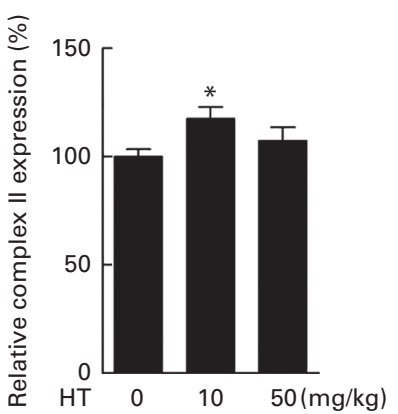

(d)

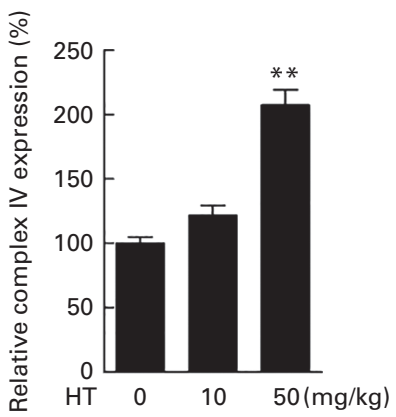

(b)

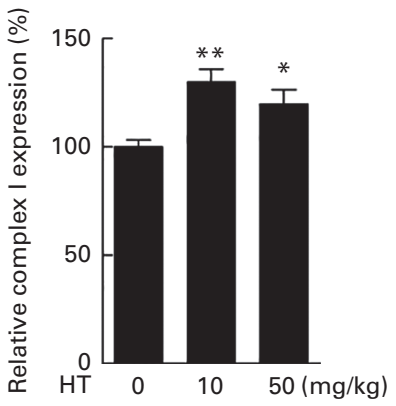

(e)

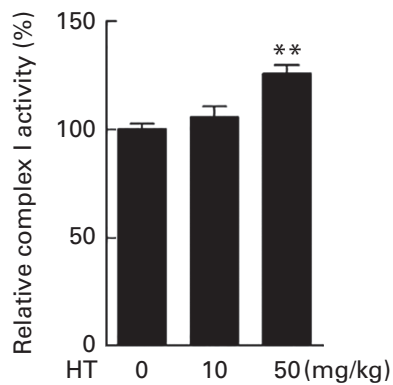

Fig. 1. Effects of hydroxytyrosol $(\mathrm{HT})$ on the expression and activities of mitochondrial complexes in the $d b / d b$ mouse brain cortex. After 8 weeks of treatment with $\mathrm{HT}$, mice were killed for brain protein and mitochondrial isolation. Protein expression levels of brain mitochondrial complex subunits were determined by the following Western blotting analysis: Western blot image (a), statistical analysis of complex I subunit (b), complex II subunit (c) and complex IV subunit (d) levels. Mitochondrial respiratory chain complex I activity was analysed spectrometrically (e). Expression of each protein was normalised to the $\beta$-actin loading control. Values are means, with their standard errors represented by vertical bars $(n 8)$. Mean value was significantly different from that of control group: ${ }^{\star} P<0.05$, ${ }^{\star \star} P<0.01$. 


\section{Results}

Effects of hydroxytyrosol on the expression and activities of mitochondrial complexes in the $\mathrm{db} / \mathrm{db}$ mouse brain cortex

The $d b / d b$ mice exhibited over obesity and hyperglycaemia relative to normal. Comparing with normal C57BL6 mice, no significant changes were observed on the expression of mitochondrial complexes in the $d b / d b$ mice brain cortex (see online Supplementary Fig. S1). After a 2-month HT supplement in $d b / d b$ mice, the expression of mitochondrial complexes I, II and IV was significantly improved (Fig. 1(a)-(d)), while the expression of complexes III and V was not affected. We also measured the activities of these complexes and found that the activity of complex I was significantly increased after HT treatment compared with the $d b / d b$ control group (Fig. 1(e)), while the activities of other complexes were not changed (data not shown).

\section{Hydroxytyrosol induced phase II antioxidant systems in the brain of $\mathrm{db} / \mathrm{db}$ mice}

The induction of phase II enzymes is another effect of HT in our previous in vitro studies. In the present study, we found that the phase II antioxidant enzymes, including HO-1 (Fig. 2(a) and (c)), SOD1 (Fig. 2(a) and (d)) and SOD2 (Fig. 2(a) and (e)), were significantly increased by HT treatment. Since SOD2 was found markedly decreased in $d b / d b$ mice compared with C57BL6 control mice (see online Supplementary Fig. S2), the major effect of HT on SOD content in the $d b / d b$ mice was assumed to sufficiently prevent the decrease in SOD2. A previous study has indicated that p62 can activate $\mathrm{Nrf2}$, a key regulator of phase II enzymes, and induce antioxidant proteins and detoxification enzymes $^{(26)}$. Thus, we measured p 62 protein expression and found a significant increase in p62 expression induced after HT treatment (Fig. 2(a) and (b)).

\section{Hydroxytyrosol inhibited protein oxidation in the brain of $\mathrm{db} / \mathrm{db}$ mice}

Protein carbonyl content is an indicator of protein peroxidation. As shown in Fig. 3, protein oxidation was effectively decreased by both low and high doses of HT supplements, suggesting that HT may contribute to reduce oxidative stress in the brain of $d b / d b$ mice.

\section{Hydroxytyrosol activated AMP-activated protein kinase pathway in the brain of $\mathrm{db} / \mathrm{db}$ mice}

The AMPK/Sirt1/PGC-1 pathway has been suggested as a mechanism for controlling mitochondrial function $(27,28)$ and Nrf2-regulated antioxidative enzymes ${ }^{(29)}$. Hence, we detected the AMPK/Sirt1/PGC-1 pathway in the brain of $d b /$ $d b$ mice. As expected, the expression of p-AMPK/AMPK (Fig. 4(a) and (b)), PGC-1 (Fig. 4(c) and (d)) and Sirt1 (a)

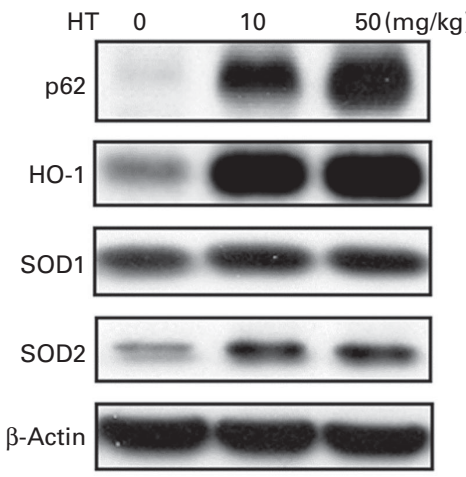

(b)

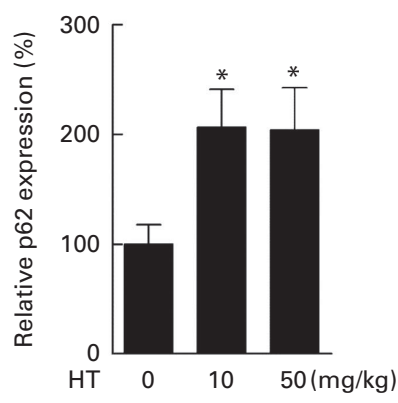

(c)

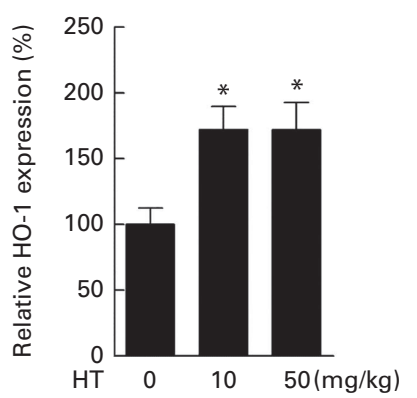

(d)

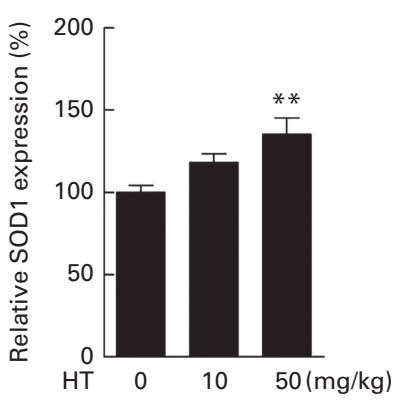

(e)

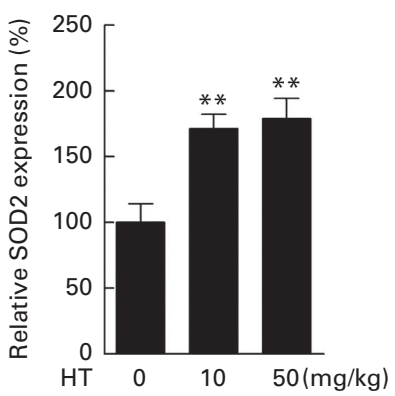

Fig. 2. Effects of hydroxytyrosol $(\mathrm{HT})$ on the phase II antioxidant system in the brain of $d b / d b$ mice. After 8 weeks of treatment with $\mathrm{HT}$, mice were killed, and the brain tissues were collected. The activation of the phase II antioxidant system was detected by the following Western blotting analysis: Western blot image (a), statistical analysis of p62 (b), haeme oxygenase 1 (HO-1) (c), superoxide dismutase (SOD)1 (d) and SOD2 (e). Expression of each protein was normalised to $\beta$-actin loading controls. Values are means, with their standard errors represented by vertical bars $(n 8)$. Mean value was significantly different from that of control group: ${ }^{\star} P<0.05,{ }^{\star \star} P<0.01$. 
(a) $\quad \mathrm{HT} \quad 0 \quad 10 \quad 50(\mathrm{mg} / \mathrm{kg})$

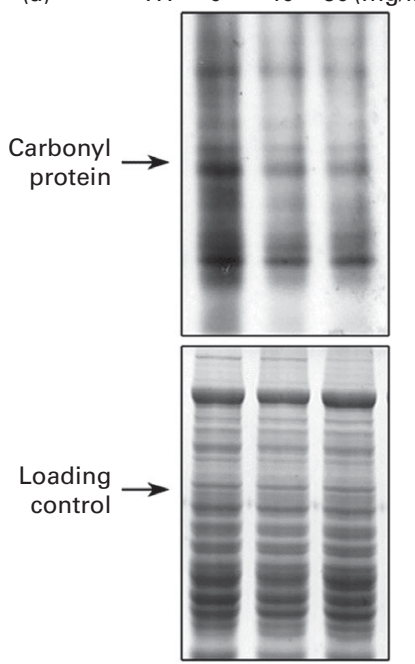

(b)

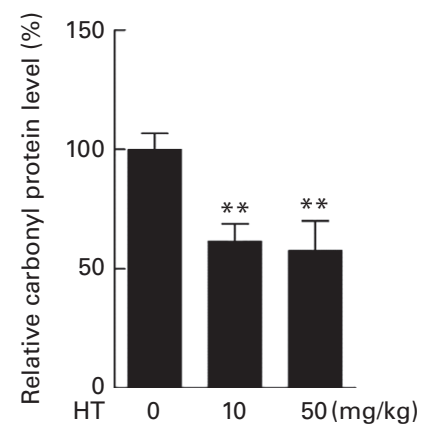

Fig. 3. Effects of hydroxytyrosol (HT) on protein oxidation in the brain of $d b / d b$ mice. After 8 weeks of treatment with $\mathrm{HT}$, mice were killed, and the brain tissues were collected. The carbonyl protein content was analysed as an indicator of protein oxidation. (a) Western blotting image and (b) statistical analysis is shown. Expression of protein was normalised to the total protein content loading controls. Values are means, with their standard errors represented by vertical bars $(n 8)$. ${ }^{* *}$ Mean value was significantly different from that of control group $(P<0.01)$.

(Fig. 4(e) and (f)) levels were all significantly increased by both low and high doses of HT supplements, suggesting that HT activated the AMPK/Sirt1/PGC-1 pathway in the brain of $d b / d b$ mice.

\section{Hydroxytyrosol improved neuronal survival of $\mathrm{db} / \mathrm{db}$ mice}

Previous study has reported that neuron loss was observed in the dorsal root ganglia of $d b / d b$ mice $^{(30)}$. In the present study, neuron loss was observed in the brain cortex of $d b / d b$ mice compared with normal C57BL6 control mice evidenced by decreased neuron factors including Arc, NMDAR1 and NGF (see online Supplementary Fig. S3). HT supplements, especially the high dose, significantly increased the mRNA levels of Arc, NMDAR1 and NGF compared with the $d b / d b$ control mice, suggesting that HT improved neuronal cell survival in the brain of $d b / d b$ mice (Fig. 5(a)-(c)).

\section{Hydroxytyrosol protected against high glucose-induced SH-SY-5Y neuronal cell damage}

Hyperglycaemia is a major factor for the complications derived from diabetes. Therefore, a toxicity model induced by high glucose in SH-SY-5Y cells was used to confirm the possible underlying mechanism of HT's neuroprotective effects in vitro. Similar to a previous report ${ }^{(31)}$, high levels of glucose ( 45 and $175 \mathrm{~mm}$ ) inhibited cell viability in a time-dependent manner within $24 \mathrm{~h}$ (Fig. 6(a)). Consistent with previous studies ${ }^{(32-35)}$, increased DCF fluorescence were observed $3 \mathrm{~h}$ after treatment with $45 \mathrm{~mm}$-glucose and plateaued after 6-24h (Fig. 6(b)). Meanwhile, pre-treatments with 2,5 and $10 \mu \mathrm{m}-\mathrm{HT}$ sufficiently increased cell viability after a $45 \mathrm{~mm}$-glucose challenge (Fig. 6(c)). Although we have previously reported that HT could be efficiently uptake in mice and reached $16 \mu \mathrm{mol} / 1(2.5 \mu \mathrm{g} / \mathrm{ml})$ at $5 \mathrm{~min}$ in serum after $50 \mathrm{mg} / \mathrm{kg}$ administration ${ }^{(25)}$, we also measured HT in culture medium with or without SH-SY-5Y cells, data suggested that HT could efficiently uptake in the cells (see online Supplementary Fig. S4). In addition, we analysed basal and uncoupled mitochondrial $\mathrm{V}_{\mathrm{O} 2}$ capacity with Seahorse analyzer (Seahorse Bioscience) (see online Supplementary Fig. S5). Normalised data indicated that the basal $\mathrm{V}_{\mathrm{O} 2}$ capacity, ATP production potential and maximal respiration were all significantly decreased by a $45 \mathrm{~mm}$-glucose treatment, while basal and maximal respiration were efficiently protected by a $10 \mu \mathrm{m}-\mathrm{HT}$ pre-treatment (Fig. 6(d)). (a)
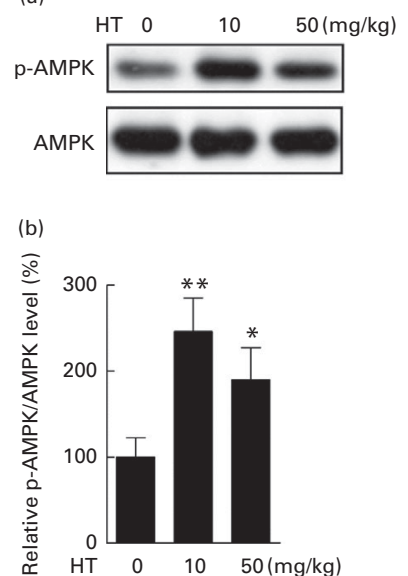

(c)

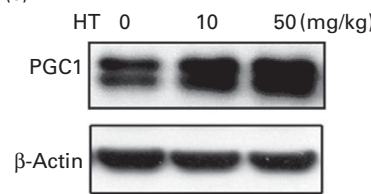

(d)

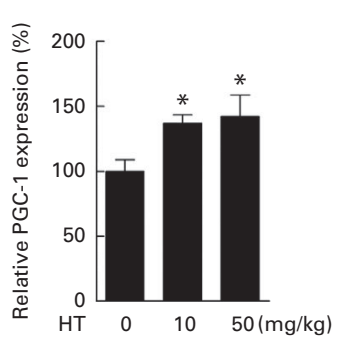

(e)

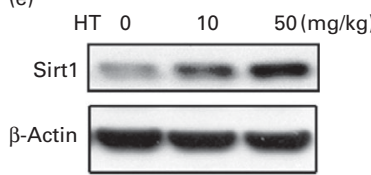

(f)

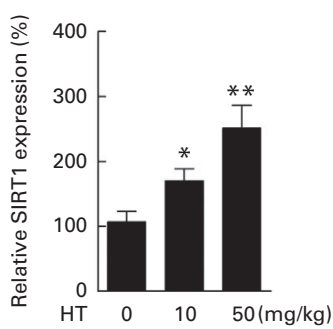

Fig. 4. Effects of hydroxytyrosol $(\mathrm{HT})$ on the AMP-activated protein kinase (AMPK) pathway in the brain of $d b / d b$ mice. After 8 weeks of $H T$ treatment, mice were killed and brain proteins were isolated for Western blotting analysis. The expression of phospho-AMPK (p-AMPK)/AMPK ((a) Western blot image, (b) statistical analysis), PPAR $\gamma$ coactivator-1 (PGC-1) ((c) Western blot image, (d) statistical analysis) and sirtuin 1 (Sirt1) ((e) Western blot image, (f) statistical analysis) were tested. The expression of each protein was adjusted to AMPK or the $\beta$-actin loading control. Values are means, with their standard errors represented by vertical bars $\left(n\right.$ 8). Mean value was significantly different from that of control group: ${ }^{\star} P<0.05,{ }^{* \star} P<0.01$. 
(a)

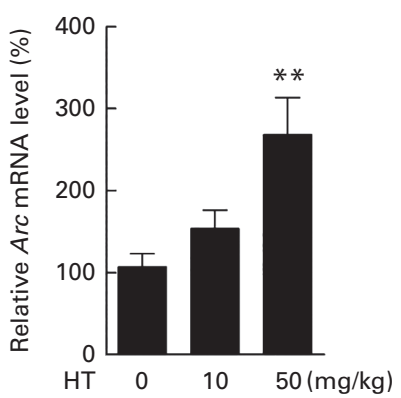

(b)

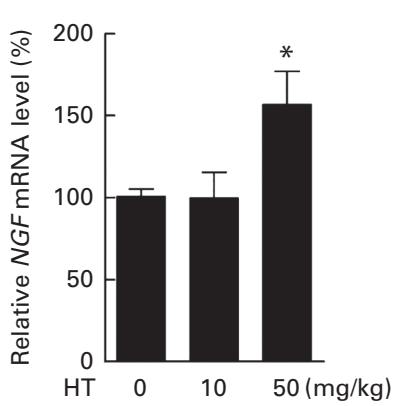

(c)

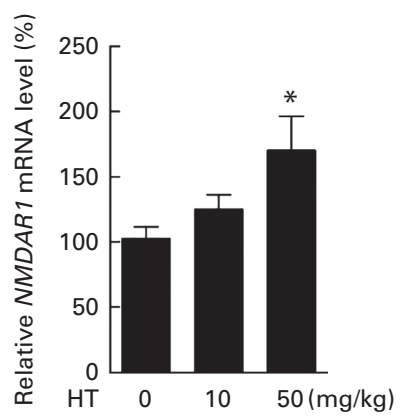

Fig. 5. Effects of hydroxytyrosol $(\mathrm{HT})$ on the mRNA expression of neuronal survival biomarkers in the brain of $d b / d b$ mice. After 8 weeks of treatment with $\mathrm{HT}$, mice were killed and total RNA was isolated. The following mRNA expression levels of neuronal factors were determined by real-time PCR as an indication of neuron survival: (a) activity-regulated cytoskeleton-associated protein (Arc) mRNA levels; (b) $N$-methyl-D-aspartate receptor 1 (NMDAR1) levels; (c) nerve growth factor (NGF) levels. Values are means, with their standard errors represented by vertical bars $(n 8)$. Mean value was significantly different from that of control group: ${ }^{*} P<0.05,{ }^{* *} P<0.01$

Hydroxytyrosol prevented high glucose-induced SH-SY-5Y cellular damage through AMP-activated protein kinase activation

Time-dependent treatment of SH-SY-5Y cells with $10 \mu \mathrm{m}-\mathrm{HT}$ increased p-AMPK levels at 10 and $30 \mathrm{~min}$, which then gradually decreased, suggesting that HT could rapidly activate the AMPK pathway (Fig. 7(a) and (b)). Meanwhile, HT treatment significantly increased the protein expression of mitochondrial complex IV and HO-1, and the induction effect was abolished by AMPK-specific inhibitor compound C (Fig. 7(c) and (d)). Although HT showed no significant induction on SOD2 protein expression, the inhibition of AMPK sufficiently reduced its content (Fig. 7(c) and (d)), suggesting the major regulatory effect of AMPK pathway on mitochondrial and antioxidative protein expression. In addition, $45 \mathrm{~mm}$-glucose treatment significantly decreased the levels of p-AMPK, while $10 \mu \mathrm{M}-\mathrm{HT}$ pre-treatment effectively restored the levels of p-AMPK
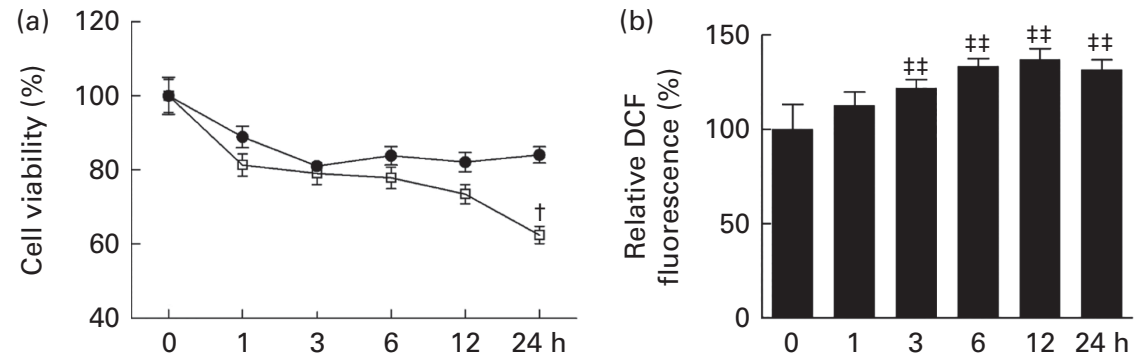

(c)
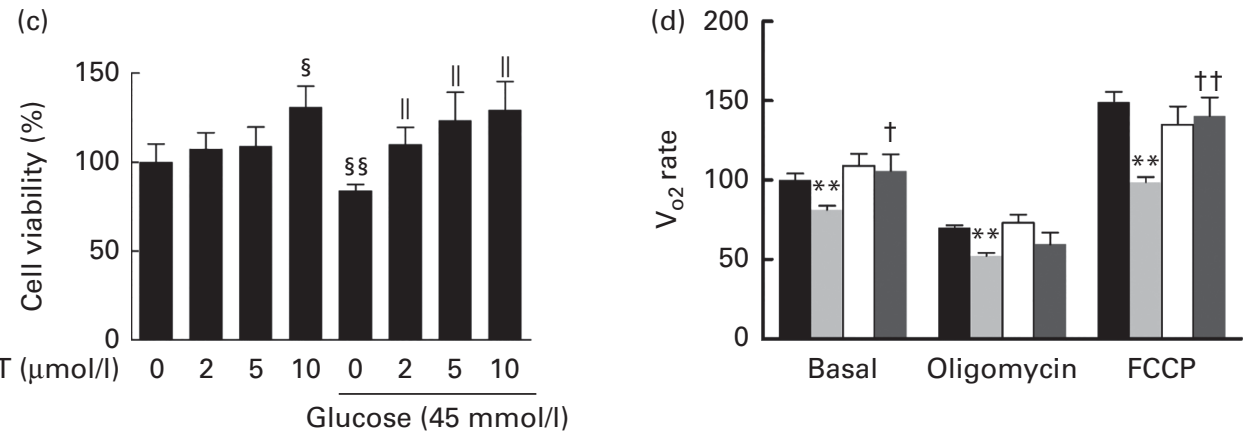

Fig. 6. Effects of hydroxytyrosol $(\mathrm{HT})$ on high glucose-induced neuronal cell damage. (a) SH-SY-5Y cells were treated with glucose at the indicated concentrations $(45 \mathrm{~mm}(-\bullet)$ and $175 \mathrm{mM}(-))$ for $1,3,6,12$ or $24 \mathrm{~h}$, and cell viability was detected by the MTT (3-(4,5-dimethylthiazol-2-yl)-2,5-diphenyltetrazolium bromide) method. (b) SH-SY-5Y cells were treated with $45 \mathrm{~mm}$-glucose for 1, 3, 6, 12 or $24 \mathrm{~h}$, and oxidative status was assessed with a 2,7-dichlorofluorescien diacetate (DCF-DA) assay. (c) SH-SY-5Y cells were pretreated with the indicated concentrations of HT $(2,5$ and $10 \mu \mathrm{M})$ for $24 \mathrm{~h}$, followed by a $6 \mathrm{~h}$ treatment with $45 \mathrm{~mm}$ glucose, and cell viability was then detected by the MTT method. (d) SH-SY-5Y cells were pretreated with $10 \mu \mathrm{M}-\mathrm{HT}$ for $24 \mathrm{~h}$, followed by a $6 \mathrm{~h}$ treatment with $45 \mathrm{~mm}$-glucose, and the basal, oligomycin-treated and carbonyl cyanide 4-(trifluoromethoxy) phenylhydrazone (FCCP) uncoupled cell $\mathrm{V}_{\mathrm{O} 2}$ capacity was detected with a Seahorse analyzer (Seahorse Bioscience). $\square$, Control; $\square$, glucose 45; $\square, \mathrm{HT}, \square, \mathrm{HT}+$ glucose 45 . Values are means, with standard errors represented by vertical bars $(n 3) .{ }^{* *}$ Mean value was significantly different from that for control $(P<0.01)$. Mean value was significantly different from that for the $45 \mathrm{~mm}$-glucose

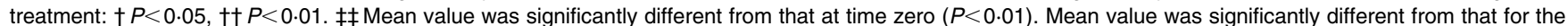
no-glucose, no-HT treatment: $\S P<0.05$, $\S \S<0.01$. ॥ Mean value was significantly different from that for the $45 \mathrm{~mm}$-glucose, no-HT treatment $(P<0.05)$. 
(a)

(b)

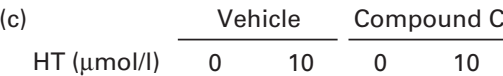
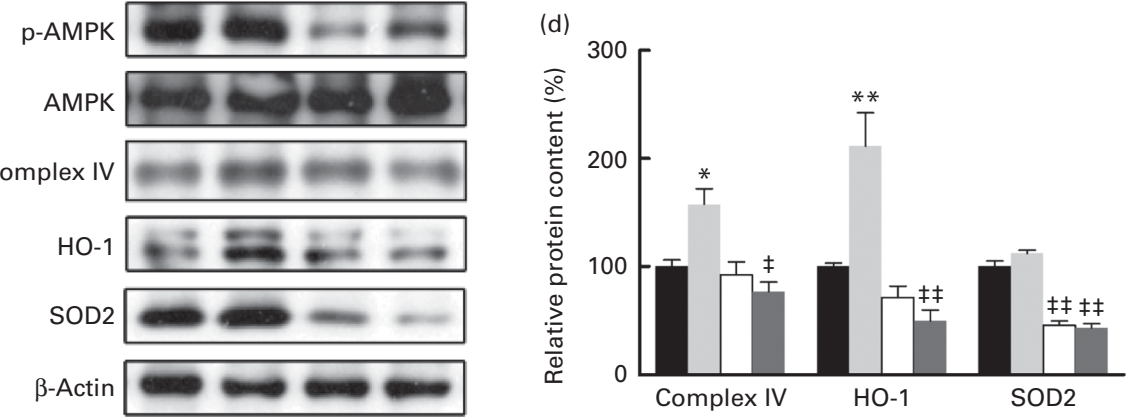

Fig. 7. Effects of hydroxytyrosol (HT) on AMP-activated protein kinase (AMPK) activation with and without glucose treatment. SH-SY-5Y cells were treated with $10 \mu \mathrm{M}-\mathrm{HT}$ for 10, 30, 60, 120, 360 min without glucose treatment, and phospho-AMPK (p-AMPK) was detected by Western blotting: (a) Western blotting image, (b) statistical analysis. SH-SY-5Y cells were pretreated AMPK inhibitor compound C with or without $10 \mu \mathrm{M}-\mathrm{HT}$ for $24 \mathrm{~h}$, protein expression of p-AMPK, AMPK, complex IV, haeme oxygenase 1 (HO-1) and superoxide dismutase 2 (SOD2) was detected by Western blotting: (c) Western blotting image, (d) statistical analysis. —, Control; $\square, \mathrm{HT} ; \square$, compound C; $\square, \mathrm{HT}+$ compound C. Values are means, with standard errors represented by vertical bars ( $n$ 3). Mean value was significantly different from that for control: ${ }^{*} P<0.05,{ }^{\star *} P<0.01$. Mean value was significantly different from that at time zero: $\dagger P<0.05, \dagger \dagger P<0.01$. Mean value was significantly different from that for the $\mathrm{HT}$-only treatment: $\ddagger P<0.05$, $\ddagger \ddagger P<0.01$.

(Fig. 8(a) and (b)), and the protective effects of HT against high glucose-induced cell toxicity were abolished when activation of AMPK was inhibited by compound C (Fig. 8(c)), indicating that AMPK pathway activation contributed to HT's protective effect.

\section{Discussion}

The Mediterranean diet is rich in olive oil and has been associated with a slower decline in cognition ${ }^{(36,37)}$. Limited studies have suggested that polyphenols with anti-oxidative properties may account for the benefits of the Mediterranean diet; however, the primary active components and mechanisms are still unclear. HT is a major polyphenol found in virgin olive oil and has been observed to have anti-inflammatory, anti-cancer and anti-obesity effects. In the present study, we are the first to link the beneficial effects of HT to the diabetic brain with its ability to improve mitochondrial function and activation of phase II antioxidant enzymes by activation of AMPK signalling.

The $d b / d b$ mice have been widely used as a model of T2DM and other metabolic conditions, such as obesity and dyslipidaemia. A previous study has also observed impaired long-term potentiation and spatial memory in $d b / d b$ mice $^{(38)}$. Furthermore, a recent study has reported central nervous system and peripheral molecular changes in diabetic $d b / d b$ mice with significant reduction in mitochondria-related molecules including complexes subunit, TCA cycle and the antioxidant SOD2, and concluded that these changes might contribute to the cognitive dysfunction in $d b / d b$ mice $^{(39)}$.
In the present study, HT treatment could significantly improve the expression levels of mitochondrial complexes I/II/IV significantly in the brain and increase the activity of complex I, the major complex in the electron transport chain and oxidative phosphorylation in the $d b / d b$ mice. In addition, HT treatment increased antioxidative enzymes, such as HO-1, SOD1 and SOD2, and decreased the protein carbonyl content in the brain of $d b / d b$ mice. Collected data suggested that HT could improve mitochondrial function and reduce oxidative stress in the brain of $d b / d b$ mice. Therefore, the mitochondrial protective effects and reduction in oxidative stress might be contributing to the increases in neuronal survival.

Previous study has shown $33 \%$ neuron loss in the brain of $d b / d b$ mice at 8-month age compared with the control ${ }^{(30)}$, which suggested that significant neuron loss may actually happen in the brain of $d b / d b$ mice. In the present study, we measured the expression of neuron markers including Arc, NMDAR1 and NGF. Arc has been associated with synaptic potentiation and the long-term consolidation of memory ${ }^{(40)}$. Arc levels are decreased in high-fat diet-fed animals and Alzheimer's diseased brains ${ }^{(41)}$. NMDAR, a neurotransmitter receptor, is a key molecule to mediate synaptic plasticity, and the activation of NMDAR is required for Arc function ${ }^{(42)}$. NGF plays a key role in the development and maintenance of sympathetic and sensory neurons as well as regulation of axonal growth and guidance. Previous studies have reported that NGF could reverse diabetes-related biochemical alterations $^{(43,44)}$. In the present study, all the three factors were significantly higher after HT treatment compared with $d b / d b$ control mice, suggesting that HT has neuroprotective effects 
(a)
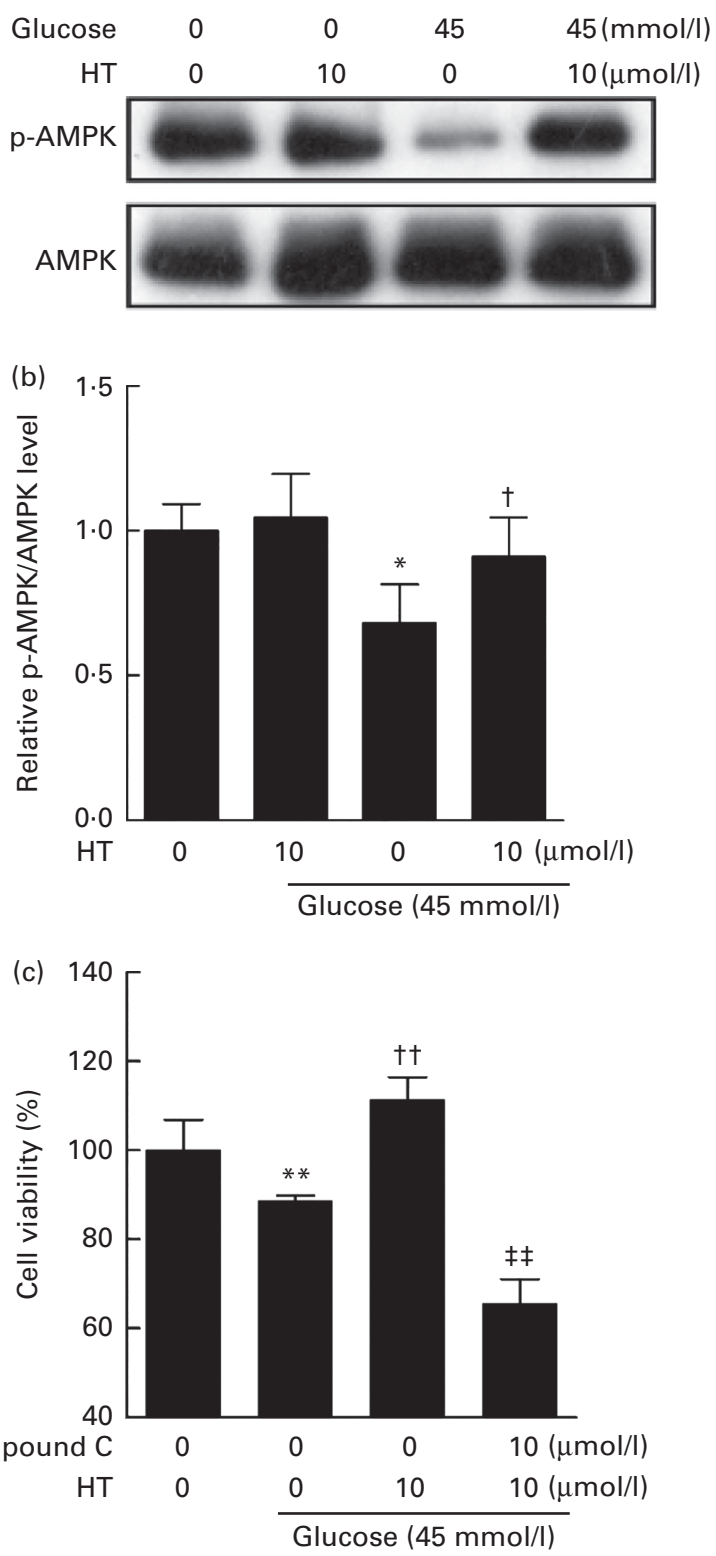

Fig. 8. Inhibition of the AMP-activated protein kinase (AMPK) pathway abolished the protective effects of hydroxytyrosol (HT). SH-SY-5Y cells were pretreated with $10 \mu \mathrm{M}-\mathrm{HT}$ followed by $6 \mathrm{~h}$ high glucose treatment. The activation of AMPK pathway was measured by Western blot: (a) Western blot image; (b) statistical analysis. Cells were treated with $10 \mu \mathrm{M}-\mathrm{HT}$ with or without compound $\mathrm{C}$ for $24 \mathrm{~h}$, followed by a $6 \mathrm{~h}$ treatment with $45 \mathrm{~mm}$-glucose, and cell viability was measured by the 3-(4,5-dimethylthiazol-2-yl)-2,5-diphenyltetrazolium bromide method (c). Values are means, with standard errors represented by vertical bars $(n 3)$. Mean value was significantly different from that for the no-glucose, no-HT treatment: ${ }^{\star} P<0.05$, ${ }^{\star \star} P<0.01$. Mean value was significantly different from that for the $45 \mathrm{~mm}$-glucose, no-HT treatment: $\dagger P<0.05$, $† \dagger P<0.01$. 㧊Mean value was significantly different from that for the $45 \mathrm{~mm}$-glucose, $10 \mu \mathrm{M}-\mathrm{HT}$, no compound $C$ treatment $(P<0.01)$. p-AMPK, phospho-AMPK.

and may potentially improve neuron survival. Whether this protection is attributed to decreased oxidative stress and the improvement of mitochondrial function or other independent mechanisms warrants further investigation.

AMPK is a key player that regulates energy metabolism and is crucial during the development and treatment of obesity, diabetes and other metabolic disorders ${ }^{(45)}$. In addition, brain development and function are closely connected to AMPK signalling, which is also involved in neurodegenerative disorders ${ }^{(45,46)}$. Impaired AMPK signalling is linked to mitochondrial dysfunction in brain cells and peripheral neuropathy in diabetes $^{(47)}$. Resveratrol is known to enhance neurite outgrowth and reduce kidney oxidative stress in diabetic rats via activating AMPK signalling ${ }^{(48)}$. It has also been reported that HT could activate AMPK to reduce intracellular ROS levels in vascular endothelial cells ${ }^{(49)}$. In this study, p-AMPK and two other regulators of AMPK signalling, Sirt1 and PGC-1, were all significantly increased in the brain of HT-treated $d b / d b$ mice. To further confirm that the AMPK pathway drives the effects of HT, we chose a high glucose-induced toxicity model in SH-HY-5Y neuroblastoma cells. Similar to previous observations in primary neurons, high glucose significantly induced oxidative stress, mitochondrial dysfunction and decreased cell viability ${ }^{(35)}$. HT pretreatment efficiently protected mitochondrial function and cell viability, and the addition of compound C, an AMPK inhibitor, abolished these HT effects. Meanwhile, HT induced overexpression of complex IV, one of the mitochondrial electron transport chain and HO-1, one of the antioxidative enzymes was also diminished by compound $\mathrm{C}$. Thus, we suggest that AMPK activation may play major roles in HT-induced mitochondrial biogenesis and phase II enzyme activation, which then protects against high glucose toxicity.

Animal studies have shown that HT can be dose dependently absorbed and excreted in urine ${ }^{(50)}$, and HT concentrations in rat plasma can reach $1.22 \mu \mathrm{g} / \mathrm{ml}$ in $5 \mathrm{~min}$ and $1.91 \mu \mathrm{g} / \mathrm{ml}$ in $10 \mathrm{~min}$ after $20 \mathrm{mg} / \mathrm{kg}$ HT oral administration ${ }^{(51)}$, suggesting that the doses of HT used in the present study should be efficiently absorbed and accumulated in vivo. However, the HT content of olive oil varies from 1.55 to $14.42 \mathrm{mg} / \mathrm{kg}^{(52)}$, thus humans with an intake of $50 \mathrm{~g}$ olive oil/d would receive $0.7 \mathrm{mg}$ of $\mathrm{HT}$ at most. The dose of $10 \mathrm{mg} / \mathrm{kg}$ used in the present study could only be achieved in humans through the use of very high dose supplements, typically supplementation studies only achieve a total intake of approximately $50 \mathrm{mg} / \mathrm{d}^{(53)}$. The present study showed that very high doses of HT improved mitochondrial function and induced phase II antioxidative enzymes, which decreased oxidative stress in brain of $d b / d b$ mice. Furthermore, the activation of AMPK signalling by HT may contribute to its protective effects. However, caution should be exercised in extrapolating these findings to lower doses of HT more typically found in humans. There remains the potential for synergistic effects of HT with other polyphenols in olive oil, which could lead to benefits in a realistic dose range suggesting that HT might be an effective agent for the prevention and treatment of diabetic complications such as brain damage.

\section{Supplementary material}

To view supplementary material for this article, please visit http://dx.doi.org/10.1017/S0007114515000884 


\section{Acknowledgements}

This work was supported by the National Basic Research Program (2015CB553602, 2014CB548200), the Fundamental Research Funds for the Central Universities (no. 08143008), the National Natural Science Foundation of China (81201023, 31370844), and the National 'Twelfth Five-Year' Plan for Science \& Technology Support (2012BAH30F03). The funders had no role in the design, analysis or writing of this article.

The authors' contributions are as follows: A. Z., J. Liu and Z. F. designed the study; A. Z., Ha. L., J. X., K. C., Hu. L., W. P. and Z. Y. conducted the research; A. Z., Y. P. and J. Long analysed the data; J. Liu and Z. F. wrote the manuscript and Z. F. had primary responsibility for the final content. All authors have read and approved the final manuscript.

The authors declare that they have no conflicts of interest.

\section{References}

1. Whiting DR, Guariguata L, Weil C, et al. (2011) IDF diabetes atlas: global estimates of the prevalence of diabetes for 2011 and 2030. Diabetes Res Clin Pract 94, 311-321.

2. Stumvoll M, Goldstein BJ \& van Haeften TW (2005) Type 2 diabetes: principles of pathogenesis and therapy. Lancet 365, 1333-1346

3. Manschot SM, Brands AM, van der Grond J, et al. (2006) Brain magnetic resonance imaging correlates of impaired cognition in patients with type 2 diabetes. Diabetes $\mathbf{5 5}$, 1106-1113.

4. Gold SM, Dziobek I, Sweat V, et al. (2007) Hippocampal damage and memory impairments as possible early brain complications of type 2 diabetes. Diabetologia 50, 711-719.

5. Qiu C, Sigurdsson S, Zhang Q, et al. (2014) Diabetes, markers of brain pathology, and cognitive cognition: the AGES-Reykjavik Study. Ann Neurol 75, 138-146.

6. Novak V, Zhao P, Manor B, et al. (2011) Adhesion molecules, altered vasoreactivity, and brain atrophy in type 2 diabetes. Diabetes Care 34, 2438-2441.

7. Moran C, Phan TG, Chen J, et al. (2013) Brain atrophy in type 2 diabetes: regional distribution and influence on cognition. Diabetes Care 36, 4036-4042.

8. Kumar A, Haroon E, Darwin C, et al. (2008) Gray matter prefrontal changes in type 2 diabetes detected using MRI. J Magn Reson Imaging 27, 14-19.

9. Brundel M, van den Heuvel M, de Bresser J, et al. (2010) Cerebral cortical thickness in patients with type 2 diabetes. J Neurol Sci 299, 126-130.

10. Zhao B, Pan BS, Shen SW, et al. (2013) Diabetes-induced central neuritic dystrophy and cognitive deficits are associated with the formation of oligomeric reticulon-3 via oxidative stress. J Biol Chem 288, 15590-15599.

11. Correia SC, Santos RX, Carvalho C, et al. (2012) Insulin signaling, glucose metabolism and mitochondria: major players in Alzheimer's disease and diabetes interrelation. Brain Res 1441, 64-78.

12. Milone M (2012) Mitochondria, diabetes, and Alzheimer's disease. Diabetes 61, 991-992.

13. Ceriello A (2003) New insights on oxidative stress and diabetic complications may lead to a "causal" antioxidant therapy. Diabetes Care 26, 1589-1596.

14. Vincent AM, Russell JW, Sullivan KA, et al. (2007) SOD2 protects neurons from injury in cell culture and animal models of diabetic neuropathy. Exp Neurol 208, 216-227.
15. Valente T, Gella A, Fernandez-Busquets X, et al. (2010) Immunohistochemical analysis of human brain suggests pathological synergism of Alzheimer's disease and diabetes mellitus. Neurobiol Dis 37, 67-76.

16. Teshima Y, Takahashi N, Nishio S, et al. (2014) Production of reactive oxygen species in the diabetic heart. Roles of mitochondria and NADPH oxidase. Circ J 78, 300-306.

17. Moreira PI, Santos MS, Seica R, et al. (2007) Brain mitochondrial dysfunction as a link between Alzheimer's disease and diabetes. J Neurol Sci 257, 206-214.

18. Visioli F, Bellomo G \& Galli C (1998) Free radical-scavenging properties of olive oil polyphenols. Biochem Biophys Res Commun 247, 60-64.

19. Stupans I, Kirlich A, Tuck KL, et al. (2002) Comparison of radical scavenging effect, inhibition of microsomal oxygen free radical generation, and serum lipoprotein oxidation of several natural antioxidants. J Agric Food Chem 50, $2464-2469$.

20. Zhu L, Liu Z, Feng Z, et al. (2010) Hydroxytyrosol protects against oxidative damage by simultaneous activation of mitochondrial biogenesis and phase II detoxifying enzyme systems in retinal pigment epithelial cells. J Nutr Biochem 21, 1089-1098.

21. Zou X, Feng Z, Li Y, et al. (2012) Stimulation of GSH synthesis to prevent oxidative stress-induced apoptosis by hydroxytyrosol in human retinal pigment epithelial cells: activation of Nrf2 and JNK-p62/SQSTM1 pathways. J Nutr Biochem 23, 994-1006.

22. Liu Z, Sun L, Zhu L, et al. (2007) Hydroxytyrosol protects retinal pigment epithelial cells from acrolein-induced oxidative stress and mitochondrial dysfunction. J Neurochem 103, 2690-2700.

23. Hao J, Shen W, Yu G, et al. (2010) Hydroxytyrosol promotes mitochondrial biogenesis and mitochondrial function in 3T3-L1 adipocytes. J Nutr Biochem 21, 634-644.

24. Wu YT, Lin LC \& Tsai TH (2009) Measurement of free hydroxytyrosol in microdialysates from blood and brain of anesthetized rats by liquid chromatography with fluorescence detection. J Chromatogr A 1216, 3501-3507.

25. Cao K, Xu J, Zou X, et al. (2014) Hydroxytyrosol prevents diet-induced metabolic syndrome and attenuates mitochondrial abnormalities in obese mice. Free Radic Biol Med 67, 396-407.

26. Komatsu M, Kurokawa H, Waguri S, et al. (2010) The selective autophagy substrate p62 activates the stress responsive transcription factor Nrf2 through inactivation of Keap1. Nat Cell Biol 12, 213-223.

27. Lira VA, Brown DL, Lira AK, et al. (2010) Nitric oxide and AMPK cooperatively regulate PGC-1 in skeletal muscle cells. J Physiol 588, 3551-3566.

28. Chau MD, Gao J, Yang Q, et al. (2010) Fibroblast growth factor 21 regulates energy metabolism by activating the AMPK-SIRT1-PGC-1alpha pathway. Proc Natl Acad Sci U S A 107, 12553-12558.

29. Mo C, Wang L, Zhang J, et al (2014) The crosstalk between Nrf2 and AMPK signal pathways is important for the anti-inflammatory effect of berberine in LPS-stimulated macrophages and endotoxin-shocked mice. Antioxid Redox Signal 20, 574-588.

30. Shi TJ, Zhang MD, Zeberg H, et al. (2013) Coenzyme Q10 prevents peripheral neuropathy and attenuates neuron loss in the $\mathrm{db}-/ \mathrm{db}$-mouse, a type 2 diabetes model. Proc Natl Acad Sci U S A 110, 690-695.

31. Kapoor R \& Kakkar P (2012) Protective role of morin, a flavonoid, against high glucose induced oxidative stress 
mediated apoptosis in primary rat hepatocytes. PLOS ONE 7 , e41663.

32. Hsu YY, Tseng YT \& Lo YC (2013) Berberine, a natural antidiabetes drug, attenuates glucose neurotoxicity and promotes Nrf2-related neurite outgrowth. Toxicol Appl Pharmacol 272, 787-796.

33. Li Y, Xu S, Zhang Q, et al. (2014) Cytotoxicity study on SHSY5Y cells cultured at high glucose levels and treated with bupivacaine. Mol Med Rep 9, 515-520.

34. Li L, Ye XP, Lu AZ, et al. (2013) Hyperglycemia magnifies bupivacaine-induced cell apoptosis triggered by mitochondria dysfunction and endoplasmic reticulum stress. J Neurosci Res 91, 786-798.

35. Russell JW, Golovoy D, Vincent AM, et al. (2002) High glucose-induced oxidative stress and mitochondrial dysfunction in neurons. FASEB J 16, 1738-1748.

36. Lourida I, Soni M, Thompson-Coon J, et al. (2013) Mediterranean diet, cognitive function, and dementia: a systematic review. Epidemiology 24, 479-489.

37. Valls-Pedret C, Lamuela-Raventos RM, Medina-Remon A, et al. (2012) Polyphenol-rich foods in the Mediterranean diet are associated with better cognitive function in elderly subjects at high cardiovascular risk. J Alzheimer's Dis 29, 773-782.

38. Li XL, Aou S, Oomura Y, et al. (2002) Impairment of long-term potentiation and spatial memory in leptin receptor-deficient rodents. Neuroscience 113, 607-615.

39. Ernst A, Sharma A, Elased K, et al. (2013) Diabetic db/db mice exhibit central nervous system and peripheral molecular alterations as seen in neurological disorders. Transl Psychiatry 3, e263.

40. Guzowski JF, Lyford GL, Stevenson GD, et al. (2000) Inhibition of activity-dependent arc protein expression in the rat hippocampus impairs the maintenance of long-term potentiation and the consolidation of long-term memory. J Neurosci 20, 3993-4001.

41. Mateos L, Akterin S, Gil-Bea FJ, et al. (2009) Activity-regulated cytoskeleton-associated protein in rodent brain is down-regulated by high fat diet in vivo and by 27 -hydroxycholesterol in vitro. Brain Pathol 19, 69-80.

42. Steward O \& Worley PF (2001) Selective targeting of newly synthesized $\operatorname{Arc}$ mRNA to active synapses requires NMDA receptor activation. Neuron 30, 227-240.
43. Unger J, Klitzsch T, Pera S, et al. (1998) Nerve growth factor (NGF) and diabetic neuropathy in the rat: morphological investigations of the sural nerve, dorsal root ganglion, and spinal cord. Exp Neurol 153, 23-34.

44. Muangman P, Muffley LA, Anthony JP, et al. (2004) Nerve growth factor accelerates wound healing in diabetic mice. Wound Repair Regen 12, 44-52.

45. Amato S \& Man H-Y (2011) Bioenergy sensing in the brain: the role of AMP-activated protein kinase in neuronal metabolism, development and neurological diseases. Cell Cycle 10, $3452-3460$

46. Ronnett GV, Ramamurthy S, Kleman AM, et al. (2009) AMPK in the brain: its roles in energy balance and neuroprotection. I Neurochem 109, 17-23.

47. Chowdhury SKR, Smith DR, Saleh A, et al. (2012) Impaired adenosine monophosphate-activated protein kinase signalling in dorsal root ganglia neurons is linked to mitochondrial dysfunction and peripheral neuropathy in diabetes. Brain 135, 1751-1766.

48. Kitada M, Kume S, Imaizumi N, et al. (2011) Resveratrol improves oxidative stress and protects against diabetic nephropathy through normalization of Mn-SOD dysfunction in AMPK/SIRT1-independent pathway. Diabetes $\mathbf{6 0}$, 634-643.

49. Zrelli H, Matsuoka M, Kitazaki S, et al. (2011) Hydroxytyrosol reduces intracellular reactive oxygen species levels in vascular endothelial cells by upregulating catalase expression through the AMPK-FOXO3a pathway. Eur $J$ Pharmacol 660, 275-282.

50. Visioli F, Caruso D, Plasmati E, et al. (2001) Hydroxytyrosol, as a component of olive mill waste water, is dose-dependently absorbed and increases the antioxidant capacity of rat plasma. Free Radic Res 34, 301-305.

51. Ruiz-Gutierrez V, Juan ME, Cert A, et al. (2000) Determination of hydroxytyrosol in plasma by HPLC. Anal Chem 72, 4458-4461.

52. Tuck KL \& Hayball PJ (2002) Major phenolic compounds in olive oil: metabolism and health effects. $J$ Nutr Biochem 13, 636-644.

53. Vazquez-Velasco M, Esperanza Diaz L, Lucas R, et al. (2011) Effects of hydroxytyrosol-enriched sunflower oil consumption on CVD risk factors. Br J Nutr 105, 1448-1452. 